\section{The role of left-right orientation in}

\section{interhemispheric matching of visual information*}

\section{MICHAEL C. CORBALLIS, AVRUM MILLER, and MICHAEL J. MORGAN McGill University, Montreal, Quebec, Canada}

Three experiments are described in which Ss matched left- or right-pointing arrowheads presented simultaneously in each visual field. In the first two, they were instructed to press one button as quickly as possible if the stimuli were the same and another if they were mirror images. In the third experiment, they pressed a single button only if the stimuli were the same on half the trials and only if they were mirror images on the other half. This experiment also included a control condition using up- and down-pointing arrowheads. In all three experiments, "same" RTs were shorter than "mirror" RTs, suggesting that left-right orientation is preserved, rather than mirror-reversed, in interhemispheric matching. This result is also contrary to previcus evidence that left-right symmetry is perceptually more salient than adjacent repetition of the same pattern.

Visual patterns that are left-right symmetrical (i.e., symmetrical about a vertical axis) often seem to be perceptually more salient than patterns exhibiting other geometrically comparable kinds of regularities. Mach (1897) provided examples which, he claimed, showed that the congruence of two forms is more immediately noticed if they are left-right mirror images than if they are up-down mirror images or if one is rotated through $90 \mathrm{deg}$ with respect to the other. More recently, Goldmeier (1937) and Rock and Leaman (1963) have confirmed that left-right symmetry is more salient than up-down symmetry. Again, Julesz (1969) has published a perceptual demonstration which shows that if the same random dot pattern is repeated in the four quadrants of a square, the repetitions are not perceived without effort and are, in fact, not normally noticed unless one is informed however, if the quadrants are mirror images about the horizontal and vertical axes, the resulting symmetries are easily perceived. Moreover, in the latter case, the left-right symmetry is more immediately perceived than the up-down symmetry. The reader can easily verify these effects by examining Julesz's figures for himself.

Mach thought that the salience of left-right symmetry was related to the bilateral symmetry of the visual system and especially of its "motor apparatus." Thus, of a figure symmetrical with respect to the median plane of the $O$, he wrote: "The right half of the figure stands in the same relation to the right half of the visual apparatus as the left half does to

*This research was supported by a grant from the National Research Council of Canada to the first author. the left half of the apparatus [Mach, 1897, p. 531." One might add to this that each half of the figure projects to the opposite cerebral hemisphere and that many of the commissures connecting the two hemispheres join mirror-image points (Cumming, 1970). This could lead to a left-right reversal of stimulus representations in interhemispheric transfer (Corballis \& Beale, 1970; Noble, 1968), which would create a natural congruence between the mirror-image halves of a left-right symmetrical figure.

This explanation is open to some objections. First, Rock and Leaman (1963) showed that the salience of left-right symmetry depends, not on the orientation of figures on the retina, but on the orientation which the $O$ perceives or imagines them to have with respect to the true vertical. For example, if the 0 tilts his head at an angle of $45 \mathrm{deg}$, a figure which is actually symmetrical about a vertical axis is perceived as more salient than one actually symmetrical about a horizontal axis. Conceivably, however, a "correction" for head tilt could be carried out before interhemispheric comparison occurs. Mach (1897) evidently considered this a plausible possibility, for he wrote "... if the plane of symmetry diverges considerably from the median plane of the observer ... the affinity of form is recognizable only by turning the figure around or by an intellectual act [p. 46, his italics]."

Secondly, Corballis and Beale ( 1970 ) have argued that interhemispheric mirror-image reversal is more likely a phenomenon of memory transfer than of perceptual transfer and, in any case, is not unambiguous. Mach (1897) notes that children often confuse bs and ds, for example, and cites this as an instance of perceptual confusion related to bilateral symmetry. More likely, however, it is a confusion of labeling; children can probably perceive the difference easily enough, but they may have trouble remembering which is which.

We present here some data which create further difficulties for a bilateral-symmetry theory. Left- or right-pointing arrowheads were flashed simultaneously on each side of a fixation point, and $O$ s decided as quickly as possible whether they were pointing in the same horizontal direction or whether they were left-right mirror images. The fixation point was precisely between the two arrowheads, so in the case of mirror-image arrowheads, the stimulation should have been symmetrical with respect to the median plane of the $O$. Thus, according to the bilateral-symmetry theory, the mirror-image arrangement should have resulted in greater perceptual salience than the arrangement in which both arrowheads pointed in the same direction; one might therefore expect "mirror" judgments to have shorter latency than "same" judgments. In fact, our experiments show the reverse to be true.

A subsidiary aim of the experiments was to compare latencies of response for different retino-cortical pathways. Each arrowhead was presented to one eye only so that transmission was either via ipsilateral pathways, if the arrowhead was in the nasal field (temporal hemiretina), or via contralateral pathways, if the arrowhead was in the temporal field (nasal hemiretina). Comparisons between the two systems have produced conflicting results. Some studies have suggested that projection via the contralateral pathways is the more efficient (e.g., Bower \& Haley, 1964; Corballis, 1964; Crovitz \& Lipscombe, 1963; Edridge-Green, 1918); others have favored ipsilateral projection (e.g., Barrel \& Parks, 1969; Handel \& Christ, 1969; McKeever \& Huling, 1970; Markovitz \& Weitzman, 1969; Overton \& Wiener, 1966). Although these investigations made use of a variety of different techniques and criteria, there is no obvious parameter which can explain the inconsistency in results.

\section{Method}

\section{EXPERIMENT 1}

Subjects. The Ss were 10 women, all between 18 and 25 years of age, with no known visual defects. Nine were right-handed, one left-handed.

Apparatus and material. Stimuli were left- and right-pointing arrowheads presented in a binocular 


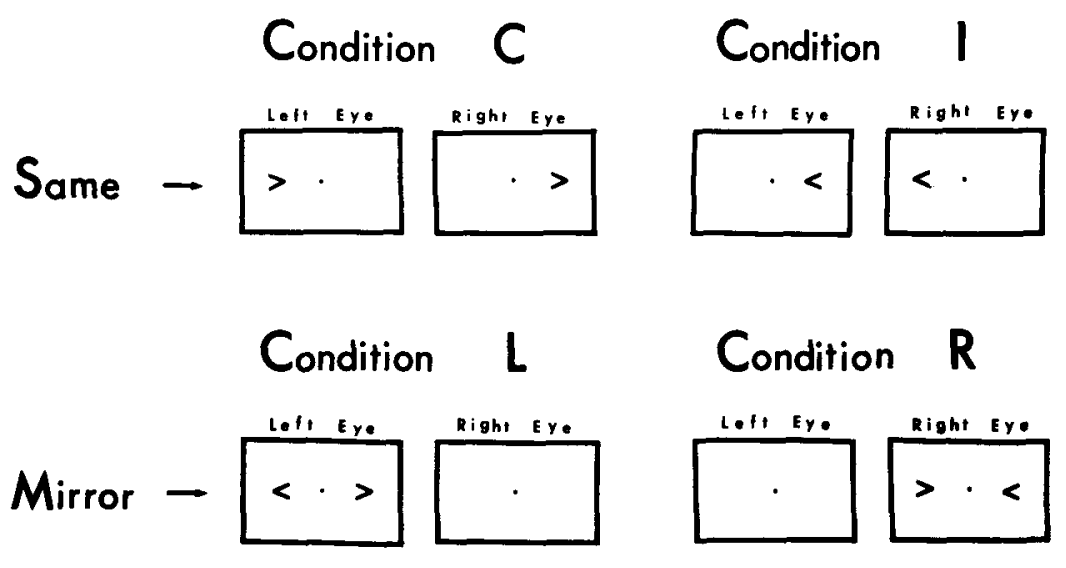

Fig. 1. Diagram illustrating the conditions of presentation used in Experiment 1. Each box shows the position of the arrowhead in the field of vision for each eye, drawn to scale. In Experiments 2 and 3, only Conditions C and I were used.

tachistoscope (Scientific Prototype Model GB). They were made by pressing black Letraset Vs onto clear slides. Each arrowhead subtended $1 \mathrm{deg}$ in width and $0 \mathrm{deg} 45 \mathrm{~min}$ in maximum height and were presented with the nearest point 2 deg from fixation. There were four conditions of presentation, illustrated in Fig. 1 . (1) In Condition C, one arrowhead was presented to the left field of the left eye and one to the right field of the right eye. This results in contralateral projection from retina to cortex. (2) In Condition I, presentation was to the left field of the right eye and to the right field of the left eye. This results in ipsilateral projection. (3) In Condition L, both arrowheads were projected to the left eye. (4) In Condition $\mathrm{R}$, both were to the right eye.

Figure 1 also illustrates the different possible orientations of the stimuli. That is, they could be "same"-both pointing left or both pointing right-or "mirror"-pointing either towards each other or away from each other. These four different possibilities were equally represented under each of the four conditions of retino-cortical transmission.

A timer was connected to the tachistoscope, its onset synchronized with stimulus onset. It was also connected to a response box, measuring 3.25 in. long $x 2$ in. wide $x$ $1.5 \mathrm{in}$. high. Two buttons were set on the top of the box, $0.5 \mathrm{in}$. from each end. The box was set on a table in front of $S$, who sat with her hands cradling the box such that the index finger of each hand rested lightly on each button. When either button was pressed, the timer stopped and displayed the time in milliseconds which had elapsed between stimulus and response. A red or a green light indicated which of the two buttons $S$ pressed.

Procedure. On each trial, a fixation point appeared for 1 sec, followed by simultaneous presentation for $100 \mathrm{msec}$ of the two arrowheads, one in each field. If the two arrowheads were pointing in the same direction, $S$ was instructed to push one button, but if they were mirror images, she was to push the other. For half of the Ss, the "same" button corresponded to the index finger of the preferred hand; for the other half, the "same" button corresponded to the finger of the nonpreferred hand. Ss were told to push the appropriate button as quickly as possible but also to make as few errors as possible. $E$ recorded the time and response choice after each trial, then reset the timer and response light system before initiating a new trial.

The first 16 trials were practice trials, consisting of each orientation condition projected under each

Table 1

Mean Reaction Times (in Milliseconds) for Each Judgment Under Each Presentation Condition in Experiment 1

\begin{tabular}{lcccc}
\hline & \multicolumn{4}{c}{ Condition } \\
\cline { 2 - 5 } Judgment & C & I & L & R \\
\hline "Same" & 549 & 513 & 539 & 532 \\
"Mirror" & 561 & 567 & 555 & 577 \\
\hline
\end{tabular}

$C=$ contralateral projection, $I=$ ipsilateral projection, $L=$ left-eye projection, $R=$ right eye projection retino-cortical condition, randomly ordered. These were followed by 64 experimental trials, also randomly ordered and balanced with respect to the different conditions.

\section{Results}

For the correct responses, reaction times were averaged for each presentation condition (see Fig. 1), judgment ("same" vs "mirror"), and orientation within judgments (i.e., leftor right-pointing within "same," inward- or outward-pointing within "mirror"). The averaged times were then subjected to analysis of variance.

There was only one significant effect; "same" RTs were shorter than "mirror" RTs [F(1,8) = 5.34, $\mathrm{p}<.05]$. RTs for each judgment are shown for each condition of presentation in Table 1. Although there seem to be slight fluctations with the presentation condition, these were not significant. There were practically no differences between orientations within judgments either in this or the following experiments, so the tabled results have been collapsed over this dimension.

Errors were very few. There were 17 altogether, 9 responses of "mirror" instead of "same," and 8 vice versa.

\section{EXPERIMENT 2}

This experiment was essentially a replication of the first, except in two respects. First, we dropped Conditions $L$ and $R$ (see Fig. 1). Secondly, we reduced stimulus exposure time from 100 to $10 \mathrm{msec}$. Our principal aim in doing this was to discover if the retino-cortical pathway would be a more critical variable with more nearly "minimal" stimulation (cf. White, 1969).

\section{Method}

Subjects. The Ss were nine women and three men, none over 25 or under 18 years of age. All were right-handed and possessed no known visual defects.

Apparatus and procedure. Apparatus and procedure were exactly as in Experiment 1, except that Conditions $\mathrm{L}$ and $\mathrm{R}$ were dropped and replaced with equal numbers of presentations of Conditions $\mathrm{C}$ and I and stimulus exposure was reduced to $10 \mathrm{msec}$. Again, there were 16 practice trials followed by 64 experimental trials.

\section{Results}

Mean reaction times for correct responses were again subjected to analysis of variance. Again, the only significant effect was that "same" was faster than "mirror" $[F(1,10)=31.44$, $\mathrm{p}<.001]$. Means for each condition are shown in Table 2. It is noteworthy that the differences between "same" 
Table 2

Mean Reaction Times (in Milliseconds) for Each Judgment Under Each Presentation Condition in Experiment 2

\begin{tabular}{llc} 
& \multicolumn{2}{c}{ Condition } \\
\cline { 2 - 3 } Judgment & $\mathrm{C}$ & I \\
\hline "Same" & 682 & 668 \\
"Mirror" & 751 & 753 \\
\hline
\end{tabular}

$C=$ contralateral projection, $I=$ ipsilateral projection

and "mirror" were much greater in this experiment than in Experiment 1; we have no explanation for this.

Errors were even fewer than in Experiment 1. The total of 15 included 7 in which Ss responded "same" instead of "mirror" and 8 in which they responded "mirror" instead of "same."

\section{EXPERIMENT 3}

The first two experiments indicated that reaction times for "same" judgments were faster than those for "mirror," contrary to an expectation that the mirror-image arrangement would be the more perceptually salient. It is conceivable, however, that our result was due more to judgmental strategy than to perceptual factors. Ss may have made the decisions sequentially, testing first for "sameness" and concluding "mirror" only if the arrowheads were not the same (although it is difficult to understand why they might do so if the mirror-image arrangement were the more salient). We tried in Experiment 3 to discourage a sequential strategy by having Ss either respond only to "same" and withhold response to "mirror" or respond only to "mirror" and withhold response to "same." With instructions to respond as quickly as possible, it would seem unlikely that Ss would test for "sameness" when they were to respond only to "mirror" unless "same" were obviously the more salient condition.

Experiment 3 also includes a control condition in which half of the Ss viewed arrowheads that were pointing up and down instead of left and right. It is well established that when Ss compare easily discriminable visual stimuli, "same" judgments have a shorter latency than do "different" judgments (Corballis, Lieberman, \& Bindra, 1968; Entus \& Bindra, 1970; $\mathrm{Nick}$ erson, 1965 ). The "same"-"mirror" difference in our experiments may represent the same phenomenon. The difference between "same" and "different" for the upand down-pointing arrowheads should provide a baseline against which to assess this possibility. We chose upand down-pointing arrowheads because they are perceptually comparable to the lateral ones hut are neutral with respect to the questiun of whether or not interhemispheric transfer involves left-right reversal.

\section{Method}

Subjects. The Ss were 10 men and 10 women, whose ages ranged between 18 and 25 years. With one exception, all Ss were right-handed. None had any known visual defect.

Apparatus and procedure. The basic equipment was the same as in the previous two experiments. Half the Ss also saw the same stimuli as in Experiment 2. For the other half, the stimuli were up- and down-pointing arrowheads made from the same Letraset Vs is the original stimuli. They were mounted so that their central vertical axes were at the same distance (2 deg $30 \mathrm{~min}$ ) from fixation as were those of the left- and right-pointing arrowheads. They were presented under exactly the same conditions of retino-cortical transmission. The fixation point again appeared $1 \mathrm{sec}$ prior to exposure, and the stimuli were projected for 10 msec.

In this experiment, Ss held the response box with only the index finger of the preferred hand placed on one of the buttons (the other button was not used). The Ss who saw the left- and right-pointing arrowheads were to press the button only if the stimuli were the same for half of the trials ( 8 practice, followed by 32 experimental triais) and only if they were left-right mirror images for the other half. Five Ss made "same" judgments followed by "mirror" ones, the other five vice versa. The Ss who saw the up-down stimuli followed exactly the same procedure, except that "mirror" for them meant up-down mirror images instead of left-right ones.

\section{Results}

Reaction times were averaged for correct responses and analysis of variance carried out. There were two significant effects; "same" reaction times were faster than "mirror" $[F(1,16)=24.75, \quad p<.001]$, and Condition $\mathrm{C}$ yielded faster reaction times than did Condition I $[F(1,16)=9.74, \quad p<.01]$. Table 3 shows these effects for each set of stimuli. It is noteworthy that although reaction times were markedly shorter for the left- and right-pointing arrowheads than for the up-down ones, individual differences were considerable and the difference was not significant $(\mathrm{F}<1)$. There were no significant interactions.

The fact that contralateral transmission system yielded the faster
Table 3

Mean Reaction Times (in Milliseconds) for Each Judgment Under Each Presentation Condition and Each Stimulus Orientation in Experiment 3

\begin{tabular}{|c|c|c|c|c|}
\hline \multirow[b]{3}{*}{ Judgment } & \multirow{2}{*}{\multicolumn{2}{|c|}{$\begin{array}{c}\begin{array}{c}\text { Left-Right } \\
\text { Orientation }\end{array} \\
\text { Condition }\end{array}$}} & \multirow{2}{*}{\multicolumn{2}{|c|}{$\frac{\begin{array}{c}\text { Up-Down } \\
\text { Orientation }\end{array}}{\text { Condition }}$}} \\
\hline & & & & \\
\hline & $\mathrm{C}$ & I & C & I \\
\hline "Same" & 361 & 391 & 418 & 433 \\
\hline "Mirror" & 451 & 455 & 493 & 529 \\
\hline
\end{tabular}

$C=$ contralateral projection, $I=$ ipsilateral projection

reaction times scarcely resolves the issue concerning the relative dominance of ipsilateral and contralateral systems, particularly since there were no differences in Experiments 1 and 2 (in fact, the differences were in the opposite direction, if anything). It is perhaps worth remarking, however, that studies in which processing time has been the major concern have generally agreed in suggesting contralateral transmission to be the faster (Bower, 1966; Lee, 1970; Woodworth, 1938).

Errors were again rare. Ss who were shown the left- and right-pointing arrowheads made 13 errors; 6 involved pressing the "same" button when the stimuli were mirror images, and 7 involved pressing "mirror" when the stimuli were the same. There were 27 errors among Ss who saw the up-down arrowheads; 7 of these were for pressing "same" for mirror stimuli and 20 of them for pressing "mirror" for same.

\section{DISCUSSION}

Our results leave no doubt that "same" was the faster judgment, and Experiment 3 suggests that this was not simply a result of sequential testing. These findings fail to support the theory which relates the salience of left-right symmetry to the bilateral symmetry of the visual system. Under conditions which might be expected to optimize the role of symmetrical interhemispheric exchange, we failed to find any evidence for the perceptual salience of a symmetrical display.

The finding that "same" judgments have shorter latency than "mirror" judgments seems to be a particular case of the general finding that "same" latencies are shorter than "different" latencies, at least when the stimuli to be compared are easily discriminable (Corballis et al, 1968; Entus \& Bindra, 1970; Nickerson, 1965). In Experiment 3, the "same"-"mirror" difference was about the same for the left- and right-pointing as for the upand down-pointing arrowheads (77 and $85 \mathrm{msec}$, respectively). However, there was a prior question as to which 
judgment, in the case of left- and right-pointing arrowheads, could be considered equivalent to "same" and which to "different." The notion of interhemispheric mirror-image reversal suggests that it should be the mirror images which effectively become "same", in the process of interhemispheric comparison; they are also "same" in the sense that both point either towards or away from the fixation point. But the results negate this interpretation and suggest that left-right orientation is preserved in interhemispheric matching.

It is perhaps not altogether surprising that left-right orientation should be preserved across the two visual fields, since reading requires that we interpret printed material in the same left-right sense in each field. This left-right equivalence between fields could be a result of postexposural left-to-right scanning. White (1969) has reviewed the evidence for such a mechanism in tachistoscopic perception. It is clear that a scanning device, sweeping left to right across a visual image in both fields, would create equivalence between the same rather than mirror-image orientations in each field. It seems likely that left-to-right scanning originates in eye movements required in reading but, with practice, becomes "semiautonomous" (Hebb, 1963). It would perhaps be interesting to study interhemispheric matching of "same" and "mirror" displays in a culture, such as the Japanese, without any strong tendency towards left-to-right scanning.

Our results contrast rather strikingly with the effects created by Julesz's (1969) published demonstration. In one of his figures, one can scarcely tell at all that random dot patterns are repeated side by side, while in the other, it is immediately obvious that adjacent patterns are mirror images. However, these effects can be explained in another way. When the mirror images are placed side by side, the symmetry is relatively easily seen because the elements to be compared are spatially contiguous along the axis of symmetry. But when identical patterns are placed together, the elements to be compared are always half a figure apart. Spatial contiguity would scarcely have been of importance in distinguishing "same" from "mirror" in our experiments, since the stimuli were extremely simple and were placed 4 deg of visual angle apart from each other.

Our results also leave unexplained the previous evidence that left-right symmetry is perceptually more salient than up-down symmetry (see introduction). Perhaps, as Rock and Leaman (1963) suggest, left-right symmetry is noticeable simply because it is such a common feature of the environment. A great many objects, both natural and man-made, exhibit at least a rough left-right symmetry. This suggests that left-right symmetry should be particularly obvious when it applies to a unitary percept, but not when it applies to the relation of different percepts to each other. This is perhaps another reason why we found no effect related to left-right symmetry, since the arrowheads of each pair were probably placed sufficiently far apart not to be seen as a unitary figure.

\section{REFERENCES}

BARREL、 J., \& PARKS, T. Hemiretinal attention under conditions of color rivalry. Perception \& Psychophysics, 1969, 5, 246-248.

BOWER, T. G. R. A local sign for depth. Nature, 1966, 210, 1081-1082.

BOWER, T. G. R., \& HALEY, L. J. Temporal effects in binocular vision. Psychonomic Science, 1964, 4, 409-410. CORBALLIS, M. C. Binocular interactions in letter recognition. Australian Journal of Psychology, 1964, 16, 38-47.

CORBALLIS, M. C., \& BEALE, I. L. Bilateral symmetry and behavior. Psychological Review, 1970, 77, 451-464,

CORBALliS, M. C.. LIEBERMAN, W., \& BINDRA, D. Discriminability versus central intermittency in same-difference judgments. Quarterly Journal of Experimental Psychology, 1968, 20,
51-61.

CROVITZ, H. F., \& LIPSCOMBE, D. B. Dominance of the temporal visual fields at a short duration of stimulation. American Journal of Psychology, 1963 . 76, 631-637.

CUMMING, W. J. K. An anatomical review of the corpus callosum. Cortex, 1970, 6 , 1-18.

EDRIDGE-GREEN, F. W. Binocular vision Journal of Physiology, 1918, 48, 37.

ENTUS, A., \& BINDRA, D. Common features of the "repetition" and "same-different" effects in reaction time experiments. Perception \& Psychophysics, 1970, 7, 143-148.

GOLDMEIER, E. Über Ahnlichkeit bei gesehnen Figuren. Psychologische Forschung, 1937, 21, 147-208.

HANDEL, S. \& CHRIST, R. E. Detection and identification of geometric forms using perhipheral and central viewing. Perception \& Psychophysics, 1969, 6, 47-49.

HEBB, $D$. $O$. The semiautonomous process: Its nature and nurture. American Psychologist, 1963, 18, 16-27.

JULESZ, B. Binocular depth perception. In W. Reichardt (Ed.), Processing of optical data by organisms and by machines. New York: Academic Press, 1969.

LEE, D. N. Spatio-temporal integration in binocular kinetic space perception. Vision Research, 1970, 10, 68-78.

MACH, E. Analysis of the sensationns. Chicago: Open Court, 1897.

MARKOVITZ, H, \& WEITZMAN, D. Monocular recognition of letters and Landolt C's in left and right visual hemifields. Journal of Experimental Psychology, 1969, 79, 187-189.

MCKEEVER, W. F., \& HULING, M. D. Left-cerebral hemispheric superiority in tachistoscopic word-recognition performance. Perceptual \& Motor Skills. $1970,30,763-766$.

NICKERSON, R, S. Response times for "same" "different" judgments. Perceptual \& Motor Skills, 1965, 20, 15-18.

NOBLE, J. Paradoxical interocular transfer of mirror-image discrimination in the optic chiasm sectioned monkey. Brain Research, 1968, 10, 127-151.

OVERTON, W., \& WIENER, M. Visual field position and word-recognition threshold. Journal of Experimental Psychology, $1966,71,249-253$.

ROCK, I., \& LEAMAN, R. An experimental analysis of visual symmetry. Acta Psychologica, 1963, 21, 171-183.

WHITE, M. J. Laterality differences in perception: A review. Psychological Bulletin, 1969, 72, 387-405.

WOODWORTH, R. S. Experimental psychology. New York: Holt, 1938.

(Acceptcd for publication May 17, 1971.) 